\title{
The Visit Before the Morning After: Barriers to Preprescribing Emergency Contraception
}

\author{
Alison Karasz, PbD \\ Nicole Tan Kirchen, MD \\ Marji Gold, MD \\ Albert Einstein College of Medicine, \\ Bronx, NY
}

Conflicts of interest: none reported

\section{CORRESPONDING AUTHOR}

Alison Karasz, PhD

Albert Einstein College of Medicine 3544 Jerome Ave

Bronx, NY 10467

akkarasz@montefiore.org

\begin{abstract}
BACKGROUND Research suggests that while advance prescription of emergency contraception (EC) increases women's access, this prescribing model is rarely used. The present study sought to explore attitudes towards EC among patients and physicians, with the goal of understanding potential barriers to advance prescription.
\end{abstract}

METHODS Qualitative, semistructured interviews were conducted with patients and clinicians in a New York City family practice clinic.

RESULTS Using qualitative interviews, we found that attitudes towards EC among patients and clinicians are complex. Both groups of participants reported favorable attitudes towards EC. There was general agreement that physicians should take a proactive role in educating patients about the method. A notable minority in each group described substantial reservations, however, especially regarding the potential for EC abuse. Such attitudes emerged mainly in the context of discussions about advance prescription. Advance prescription was viewed as greatly facilitating access to EC, but some patients and clinicians feared that ready access would encourage irresponsible sex. Some participants condoned the occasional, accidental, or emergency use of EC; however, habitual use, or the plan not to plan for sex, was viewed as morally indefensible.

CONCLUSION Findings suggest that even when attitudes towards EC are generally favorable, some physicians and patients have substantial reservations about advance prescription. Education and dialogue are needed to overcome these reservations.

Ann Fam Med 2004;2:345-350. DOI: 10.1370/afm. 105

\section{INTRODUCTION}

$\longrightarrow$ mergency contraception (EC), often known as the morning-after pill, has been available in the United States for many years. It is widely prescribed in Europe, and has been shown to be safe and effective in preventing pregnancy. Yet EC is underprescribed and underused in the United States. ${ }^{1}$ Though the lack of awareness of EC is a barrier to wider use, ${ }^{2}$ evidence indicates that physicians with good knowledge of the method routinely underprescribe $\mathrm{EC}_{1}{ }^{3}$ whereas patients who know about EC often fail to use it when needed. ${ }^{4}$ Furthermore, opinion surveys generally find favorable views of the method among both physicians and patients. $^{5-7}$ Thus, neither the lack of knowledge nor attitudes-at least as measured superficially - offer an adequate explanation of low rates of prescribing and use. . $^{4,9}$

One factor identified as a barrier to increased EC use is the prescribing model typically used by physicians. Few physicians educate patients about EC or assess need for EC during routine health or contraceptive-counseling visits. ${ }^{3,10,11}$ Most prescribe EC on an emergency basis, when a patient 
requests it after an act of unprotected intercourse. In addition to preventing EC from reaching many patients who may not know enough about EC to ask for it, the emergency model constrains access in other ways. For example, it discourages requests among patients who fear their physician's disapproval and results in limited access to EC when the clinic is not open-the time when most sexual activity occurs. ${ }^{8}$

In places where EC is not available over-the-counter, an alternative to the emergency model is advance prescription. A number of studies have found that women who are given advance prescriptions are more likely to use EC after an incident of unprotected intercourse. ${ }^{2,12}$ Furthermore, advance prescription does not increase rates of unprotected intercourse. ${ }^{12,13}$ Even so, advance prescription is extremely rare in the United States, as it is elsewhere. ${ }^{8}$ The attitudinal barriers that might have an impact on clinicians' willingness to provide advance prescription, as well as patients' willingness to request it, have been little investigated.

The current study explored attitudes towards EC among patients and family practice clinicians at an inner-city health center in the northeastern part of the United States. In addition to gauging general attitudes towards EC, the study sought to understand patients' and clinicians' attitudes towards several prescribing models ranging in restrictiveness from the emergency model to advance prescription. The overall goal of the study was to identify possible attitudinal barriers to advance prescription that could be addressed in an educational intervention for clinicians and patients.

Most studies of attitudes and experiences associated with EC use quantitative survey methods; qualitative methods have rarely been used. 9,14,15 Yet compared with questionnaires, qualitative methods may be better suited to the exploration of attitudes, which are complex, contradictory, or context dependent. ${ }^{16,17}$ The present study used a qualitative approach to explore patients' and clinicians' attitudes towards EC.

The study was approved by the Institutional Review Board at Montefiore Medical Center.

\section{METHODS}

\section{Sample and Setting}

Participants were recruited from several primary care clinics serving a multiethnic, low-income population in the Bronx, NY. A convenience sample of 38 patients waiting for their appointments participated in the study. English-speaking women older than 16 years were eligible to participate. A sample of 25 primary care clinicians was recruited from 2 family medicine clinic sites.

The sample size was not determined in advance.
In keeping with the principals of qualitative research, data collection among patients and clinicians continued until it became evident that no new themes were emerging in the data, a point known as theoretical saturation. ${ }^{17}$

\section{Data Collection}

\section{Patients}

Twelve medical students assisted with the study during their third-year family medicine rotations. Women were approached by interviewers in the waiting rooms of the health centers. After signing an informed consent document, women participated in a semistructured interview. The investigators assumed that most patients were unfamiliar with $\mathrm{EC}_{i}$ therefore, the interview began with an educational component during which interviewers described the indications, effectiveness, and risks of EC. The interview then focused on attitudes towards EC, opinions regarding the various prescribing models, and current contraceptive use. Patient interviews occurred in private offices and lasted 20 to 30 minutes.

Interviewers were trained by the researchers to take extensive notes during the patient interviews and to prepare transcriptions immediately after the interviews. Though inferior to the use of taped transcripts, which was deemed impracticable in this setting, this transcription method has been used successfully in previous studies. ${ }^{18,19}$

\section{Clinicians}

Medical students, a college student, and a master's level intern conducted interviews with clinicians. All clinicians at the 2 sites were approached in rooms used by preceptors. Most interviews were conducted in examination or conference rooms. Six were conducted by telephone. The interview focused on themes similar to those of the patient interview, except that it also included questions about participants' history of prescribing EC. Clinician interviews lasted 30 to $45 \mathrm{~min}$ utes. These interviews were taped and transcribed.

\section{Analysis}

A preliminary coding list focusing on themes related to barriers to the prescription and use of EC was derived after an intensive review of initial interviews by 2 of the authors $(\mathrm{AK}, \mathrm{MJ})$. All authors coded a portion of the data using the preliminary code list. The list was then adapted to make it fit the data more closely, and another group of interviews was coded to determine the final version of the coding list. In a last step, 2 authors $(\mathrm{AK}, \mathrm{MJ})$ coded all of the interviews. To enhance reliability, the third author (NTK) also coded a portion of interviews. The few discrepancies in cod- 
ing were resolved by discussion among the 3 authors. The coded data were entered into N-Vivo, a qualitative data analysis computer program that facilitates the rapid retrieval of coded data. Blocks of coded data were retrieved and summarized in the preparation of results.

\section{RESULTS}

\section{Patients}

No patient approached refused to participate in the study. See Table 1 for a description of the sample. As an informal gauge of unmet need, we asked patients about current use of conventional contraceptives. Contraceptive failure and nonuse of contraceptives were common (Table 2).

In this sample, knowledge of EC was very low. More than one half of the participants $(n=25)$ had never heard of EC. Of those who had heard of EC, about one-half had basic knowledge. One person had previously used EC.

In general, attitudes towards EC were extremely favorable. Once the medication was described, most women (29/38) were enthusiastic: "Sounds great! Where can I get it?"

Tempering favorable attitudes towards EC were

\begin{tabular}{lc|}
\hline \multicolumn{2}{l}{ Table 1. Characteristics of the Sample } \\
\hline Characteristics & Value \\
\hline Patients & Median Number \\
Age, years & $28(16-61)$ \\
Education, years & $12(9-18)$ \\
Income, \$ (in thousands) & $30(8-120)$ \\
& Percent \\
Married & 29 \\
Self-reported ethnicity & \\
Hispanic & 48 \\
Black & 26 \\
White & 26 \\
Clinician specialty & Number \\
Nurse practitioner & 1 \\
Physician & 24 \\
Attending & 9 \\
Resident & 15 \\
\hline
\end{tabular}

Table 2. Patients' Experiences of Contraceptive Lapses

\begin{tabular}{lc}
\hline Lapse & Percent \\
\hline Intercourse without attempting contraception & \\
At least once in past year & 92 \\
At least once in past month & 42 \\
Experienced broken condom & 23 \\
\hline
\end{tabular}

women's concerns about the medical side effects of using EC. When asked what concerns they had about EC for either themselves or others, more than one half of the women were concerned about long-term side effects. Many of these concerns echoed common beliefs about the side effects of conventional oral birth control methods, such as hair loss and infertility.

Only 2 women objected to EC because of potential abortifacient effects. When we attempted to gauge other, more subtle moral concerns by asking patients to describe situations in which EC would be appropriate or inappropriate, however, one third $(\mathrm{n}=12)$ suggested that EC would be inappropriate for women who used it frequently. These women believed that there was a right way to use EC, defined as an infrequent emergency, such as rape or a broken condom; and a wrong way, defined as a regular or frequent reliance on the method. As one woman stated, "EC should be available to those that make mistakes, not a regular contraception."

Some suggested that unplanned sex or sex that did not include precoital contraception was irresponsible or immoral. Whereas an occasional emergency use of EC was acceptable_-"Nobody's perfect, everybody makes mistakes"-planning to use EC meant failing to plan for sex: "[EC should not be] for people who just want pleasure and don't think about consequences." One woman who believed that EC was immoral because "sex is for having babies" used condoms as a regular form of birth control. Such contradictions suggest that for some women, EC is not real contraception, and that the idea of a postcoital method of birth control is morally problematic.

\section{EC Prescription Models}

We talked with patients about their reactions to 3 prescribing models. With model 1 , the emergency model, the physician does not routinely initiate discussions of EC, but provides a prescription to women who ask for them. Model 2, the routine counseling model, is more proactive: the physician routinely counsels the patient about EC and tells her how to obtain the medication in case of need. Model 3 is the advance prescription, or medicine cabinet, model in which patients are routinely counseled and offered a prescription or an EC packet during the office visit.

When we asked for reactions to the 3 prescribing models, we found that most patients (28 of 36) preferred a proactive role for the physician. Most said that physicians should raise the issue of EC and provide routine counseling. By contrast with physicians (see below), who were concerned about identifying women at risk, many women noted that all heterosexually active women should receive counseling about the method. Some noted that it could be uncomfortable 
for patients to bring up the topic themselves. Several women wondered why they had not been informed about EC previously.

Most participants favored the advance prescription model. Women described several potential barriers to accessing EC in the absence of an advance prescription, including the difficulty of obtaining EC after clinic hours, as well as embarrassment at requesting a prescription. These patients noted the superior convenience of the advance prescription model: "I prefer her to give it to me right here. Why I got to call her for it? You never know when it's gonna pop."

Among those not in favor of the advance prescription model, however, a major objection was that it might increase sexual risk taking. Often the objections to advance prescription were strongly moral in tone. Many women thought that having EC at home could increase sexually irresponsible behavior: "If I had the prescription at home, I'd be afraid that I'd be more irresponsible. It'd be too tempting ... I'd be more likely to take a chance...."

Moral concerns about advance prescription were often related to the view, described above, that easily available postcoital contraception could rob irresponsible sex of its necessary consequences: "A woman might feel embarrassed (to call the doctor after unprotected sex) ... but I think that if they don't want to be pregnant or have an abortion, they would call the doctor. They have to take responsibility for their actions."

\section{Clinicians}

No clinician refused to participate in the study. Table 1 displays the characteristics of the clinician sample. Most clinicians in the sample had knowledge of EC sufficient to prescribe it, at least with the help of drug reference information, but only about one fourth of clinicians in the sample said they prescribed EC often. Table 3 displays the clinicians' knowledge and prescribing patterns. General attitudes towards EC were favorable; concerns arose only in the context of discussions about the different prescription models.

During the interviews, most clinicians suggested that the most restrictive prescribing model, model 1,

\section{Table 3. Clinicians' Knowledge of and Prescribing} Patterns for Emergency Contraception

\begin{tabular}{ll}
\hline Characteristics & No. \\
\hline Knowledgeable about emergency contraception & 10 \\
Model most used & 11 \\
Model 1 emergency model & 4 \\
Model 2 routine counseling model & 4 \\
Model 3 medicine cabinet model & 5 \\
Routinely discuss emergency contraception with patients & \\
\hline
\end{tabular}

was inadequate. Patients' lack of knowledge as well as embarrassment in bringing up the topic were frequently mentioned as problems associated with model 1 . A few clinicians were concerned that patients might be offended by the routine discussion of EC associated with model 2. Others worried that talking about EC sent the wrong message by implying that routine contraception might not work well: "I'm more focused on the primary preventive method they're going to use first, and getting them to start using that, rather than thinking or assuming it's going to fail."

A concern among many participants was the perceived need to identify which patients should be counseled about EC: "I discuss contraception with everyone, but I only discuss emergency contraception with those at risk. I'm not sure this makes sense."

Though almost all of the 25 clinicians in the sample agreed on the issue of routine counseling, there was much more variation in attitudes towards advance prescribing. Most clinicians said that they would like to use the advance prescription model more consistently, but 10 had serious reservations.

The concern expressed most frequently about the advance prescription model was that making EC more accessible to patients would increase rates of unprotected intercourse. Even clinicians who were in favor of advance prescription believed that it might increase sexual risk taking, making patients vulnerable to sexually transmitted infections. Concerns about exposure to sexually transmitted diseases often merged in subtle ways with moral concerns. Unlike patients, most physicians were hesitant to state moral concerns bluntly. Yet among some clinicians, as among patients, we noted that the notion of a postcoital method of contraception was troubling. Several clinicians distinguished between EC and pregnancy prevention, as if EC were not a form of prevention. One clinician, while clearly understanding the difference between EC and mifepristone, noted: "If you use contraception, it means for me that you are preventing things. But [if you use emergency birth control], you did not prevent."

Some clinicians suggested that women who chose EC as a regular method did not deserve to have EC prescribed in advance, suggesting that planning to use EC meant failing to plan for contraception: "An inappropriate candidate would be somebody who consistently turns down other methods of contraception, but then feels like they can take EC on a regular basis."

Some clinicians believed that because using EC meant having intercourse without thinking of the consequences, it was the obligation of the physician to provide (unpleasant) consequences for the patient: "I do not agree with advanced prescription.... If the patient has to call in for a prescription, it's one more step. The 
clinician [can] give her a lecture, or more education, [rather than her] just going to the cabinet and getting the pill."

A second group, less punitive in tone, believed they should follow up with patients after acts of unprotected sex. "If they page you [for a prescription for EC] ... you have a chance to know what is going on, other issues that might be related to this unprotected sex episode."

Finally, a few were concerned that EC might be misused or that patients would not understand how to use it: "If I give a prescription, they might give it to others. They might not adhere to the instructions. They might be pregnant and still take them." Another said, "I don't know if I would rely on the patient's judgment as to when to pop the pill."

As did patients, clinicians differed on the issue of which patients would be appropriate candidates for advanced prescription. Some focused on advance prescription for patients who were at risk, proposing that so-called irresponsible women were the best candidates: "If I am not confident that they will come to me and ask me when they want it, I prefer prescribing it to them in advance."

More commonly, however, clinicians noted that those who might have higher need were less-appropriate candidates for advanced prescription: "If I had patients who were in stable relationships and were at a certain age ..., I would feel comfortable with [advance prescription]. But if ... they were at risk for other things, I wouldn't be as comfortable."

A few believed that it was the obligation and privilege of the clinician, not the patient, to exert control over contraception. Patients who tried to be in control did not deserve EC: "Who would be an inappropriate candidate for EC? A patient I don't know. A patient who's not really responsible about contraception. Someone who is taking care of her own contraceptive problems. She just calls me when she wants it."

\section{DISCUSSION}

\section{Patients}

Overall, women patients attending this inner-city clinic were enthusiastic about the potential of EC to address important problems in their lives, including unplanned pregnancy, abortion, and women's lack of control over sexuality and reproduction. Women did raise a number of medical concerns, many of which reflected myths associated with precoital oral contraceptives. Moral concerns were also widespread in our patient sample and were usually associated with the notion of repeated use of EC. While acknowledging that everybody makes mistakes, many believed that the persistent or deliber- ate use of EC—a plan not to plan for sex-was irresponsible. Patients disagreed about which women were the most appropriate candidates for EC, with some believing that women who were frequent users did not deserve EC, and others believing that such irresponsible women are precisely those for whom EC would be useful. Both attitudes stigmatize EC use.

When asked about their preferences for prescribing models, women were overwhelmingly in favor of a proactive role for the physicians. Regarding advance prescription of EC, many women thought this prescribing model would increase the likelihood of their taking EC. One half of the sample, however, had reservations about advance prescription of EC, most of which related to concerns about habitual or repeated use.

\section{Clinicians}

We found many similarities in patients' and clinicians' attitudes towards EC. Most clinicians showed favorable overall attitudes towards EC and advocated a proactive role for the clinician that included routine counseling, though few were actually using this model at the time of the interview.

Serious reservations about the advance prescription model emerged in a substantial minority. These reservations appeared to be related to concerns, also found in other studies, 9,14 about abuse of EC. Many clinicians were concerned that advance prescription, by easing access, would facilitate repeated use.

As has been noted by the scholar Linda Gordon, the language of sexual risk taking in modern medical contexts is often a highly moralized, though rarely explicitly, moral discourse. ${ }^{20}$ Some clinicians expressed reluctance to offer advance prescription because of a concern that facilitated access to EC might increase a woman's risk for sexually transmitted diseases, although these same clinicians prescribed many other contraceptive methods-birth control pills, injectable contraceptives, intrauterine devices - that do not offer protection against infection. This inconsistency suggests that a major, if tacit, objection to EC has more to do with moral concerns about postcoital methods of contraception than with actual safety concerns. It is striking that several participants suggested that patients who did not have a more regular (ie, precoital) method of contraception were inappropriate candidates for advance prescription.

Some physicians proposed unrealistic strategies for controlling unprotected sexual intercourse among patients. Although only a few seemed punitive in their tone, many believed that it was their responsibility to counsel patients after incidents of unprotected sex. These physicians did not recognize the frequency of unprotected intercourse among their patients or the 
likelihood that embarrassing lectures or counseling could prevent patients from contacting the physician after unprotected sex.

\section{Implications}

Some of the barriers to increasing the prescription and use of EC in our sample could be easily addressed through a simple educational program. For example, studies show that making EC available through advance prescription increases EC use after unprotected sex but does not increase frequency of unprotected intercourse Sharing this information with patients and physicians might facilitate prescribing and use. Because some clinicians are concerned about sending a message that it is okay to engage in unprotected sex, it may be appropriate to present these data to physicians in the context of a harm-reduction approach.

Another barrier that could be addressed through education is the perception among clinicians that unprotected intercourse is abnormal. The label emergency contraception might contribute to this misperception by suggesting that contraception failure is an unusual, abnormal event. Evidence, however, indicates that it is extremely common. ${ }^{21}$ The normalization of contraceptive lapses can do much to decrease the shame patients feel about incidents of unprotected intercourse, facilitating communication between physicians and patients.

A final barrier to advance prescription is the view among some patients and clinicians that postcoital contraception is morally inferior to precoital contraception. Many physicians may feel discomfort in acknowledging that moral values underlie their professional judgments. ${ }^{20}$ Thus, in addressing this issue in the context of a physician education program, open discussion and audience participation might be more helpful than a conventional lecture format. In presenting the findings from this study, we often find that open discussion on this point generates heated discussions. Clinicians report that these discussions lead them to question their own unarticulated assumptions and to rethink their strategies in prescribing EC. Once physicians feel comfortable with the notion that postcoital contraception is real prevention, they may be able to take an active role in legitimizing the method for their patients.

To read or post commentaries in response to this article, see it online at http://www.annfammed.org/cgi/content/full/2/4/345.

Key words: Contraception; family planning services; health knowledge, attitudes, practice; women's health; qualitative research

Submitted April 29, 2003; submitted, revised, September 3, 2003; accepted October 14, 2003.

\section{References}

1. Glasier A. Safety of emergency contraception. J Am Med Womens Assoc. 1998; 35:219-221.

2. Blanchard K. Improving women's access to emergency contraception: innovative information and service delivery strategies. J Am Med Womens Assoc. 1998;53(5 Suppl 2):238-241.

3. Gold MA, Schein A, Coupey SM. Emergency contraception: a national survey of adolescent health experts. Fam Plann Perspect. 1997; 29:15-24.

4. Lewis C, Wood C, Randall S. Unplanned pregnancy: is contraceptive failure predictable? Br J Fam Plann. 1996;22:16-19.

5. Brown JW, Boulton ML. Emergency contraceptive pill dispensation in Michigan Title X clinics. Am J Public Health. 1998;88:1380-1383.

6. Muia E, Ellertson C, Lukhando M, Elul B, Clark S. Emergency contraception in Nairobi, Kenya: knowledge, attitudes and practices among policy makers, family planning providers and clients, and university students. Contraception. 1999;60:223-232.

7. Smith B, Gurney E, Aboulela L, Templeton A. Emergency contraception: a survey of women's knowledge and attitudes. Br J Obstet Gynaecol. 1996; 103:1109-1116.

8. Ellertson C, Shochet T, Blanchard KT, Russell J. Emergency contraception: a review of the programmatic and social science literature. Contraception. 2000;61:145-186.

9. Ziebland S. Emergency contraception: an anomalous position in the family planning repertoire? Soc Sci Med. 1999;49:1409-1417.

10. Weisberg E, Fraser IS, Carrick SE, Wilde FM. Emergency contraception: general practitioner knowledge, attitudes and practice in New South Wales. Med J Aust. 1995;162:136-138.

11. Delbanco SF, Stewart FH, Koenig JD, et al. Are we making progress with emergency contraception? Recent findings on American adults and health professionals. J Am Med Womens Assoc. 1998;53:242-246.

12. Glasier A, Baird D. The effects of self-administering emergency contraception. N Eng J Med. 1998;339:1-4.

13. Skibiak JP, Ahmed Y, Ketata M. Testing Strategies to Improve Access to Emergency Contraception Pills: Prescription vs Prophylactic Distribution. Nairobi: Population Council; 1999.

14. Harper C, Ellertson C. Knowledge and perceptions of emergency contraception among a college age population: a qualitative approach. Fam Plann Perspect. 1995;27:149-154.

15. Ziebland S, Graham A, McPherson A. Concerns and cautions about prescribing and de-regulating emergency contraception: a qualitative study of GPs using telephone interviews. Fam Pract. 1998;15:449456.

16. Crabtree B, Miller W. A qualitative approach to primary care research: the long interview. Fam Med. 1991;23:145-151.

17. Miller W, Crabtree BF. Research methodology qualitative analysis: how to begin making sense. Fam Pract Res J. 1994;14:289-297.

18. Karasz A, Anderson M. The vaginitis monologues: women's experiences of vaginal complaints in a primary care setting. Soc Sci Med. 2003;56:1013-1021.

19. Karasz A, Sacajiu G, Garcia N. Conceptual models of psychological distress among low-income patients in an inner-city primary care clinic. J Gen Intern Med. 2003; 18:475-477.

20. Gordon L. Teenage pregnancy and out of wedlock birth: morals, moralism, experts. In: Brandt A, Rosen P, eds. Morality and Health. New York, NY; Routledge; 1997.

21. Rowlands S. A two-year audit of emergency contraception in a general practice. Br J Fam Plann. 1991;17:23-24. 